\title{
Review
}

\section{Transmission of severe acute respiratory syndrome-coronavirus-2 (SARS-CoV-2) through infant feeding and early care practices: A systematic review}

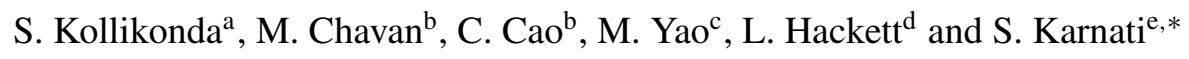 \\ ${ }^{a}$ Department of Obstetrics and Gynecology, Women's Health Institute, Cleveland Clinic, Cleveland, OH, USA \\ ${ }^{\mathrm{b}}$ Case Western Reserve University School of Medicine, Cleveland, OH, USA \\ ${ }^{\mathrm{c}}$ Department of Qualitative Health Sciences, Cleveland Clinic, Cleveland, OH, USA \\ ${ }^{\mathrm{d}}$ Floyd D Loop Alumni library, Cleveland Clinic, Cleveland, OH, USA \\ e Department of Neonatology, Cleveland Clinic Children's Hospital, Cleveland, OH, USA
}

Received 21 March 2021

Revised 31 May 2021

Accepted 3 June 2021

\begin{abstract}
.
BACKGROUND: Perinatal practices such as breast-feeding, kangaroo mother care, rooming-in, and delayed cord clamping have varied by institution during the COVID-19 pandemic. The goal of this systematic review was to examine the success of different practices in preventing viral transmission between SARS-CoV-2 positive mothers and their infants.

METHODS: Electronic searches were performed in the Ovid MEDLINE, Ovid Embase, Cochrane Library, EBSCOhost CINAHL Plus, Web of Science, and Scopus databases. Studies involving pregnant or breastfeeding patients who tested positive for SARS-CoV-2 by RT-PCR were included. Infants tested within 48 hours of birth who had two tests before hospital discharge were included. Infants older than one week with a single test were also included.

RESULTS: Twenty eight studies were included. In the aggregated data, among 190 breastfeeding infants, 22 tested positive for SARS-CoV-2 (11.5\%), while 4 of $152(2.63 \%)$ among bottle-fed (Fisher's exact test $p=0.0006)$. The positivity rates for roomed in infants $(20 / 103,19.4 \%)$ were significantly higher than those isolated $(5 / 300,1.67 \%)(P<0.0001)$. There was no significant difference in positivity rate among infants who received kangaroo care ( $25 \%$ vs $9 \%, p=0.2170)$, or delayed cord clamping $(3.62 \%$ vs $0.9 \%, p=0.1116)$.

CONCLUSIONS: Lack of robust studies involving large patient population does not allow meaningful conclusions from this systematic review. Aggregated data showed increased positivity rates of SARS-CoV-2 among infants who were breast fed and roomed-in. There were no differences in SARS-CoV-2 positivity rates in infants received skin to skin care or delayed cord clamping.
\end{abstract}

Keywords: Breast feeding, COVID-19, delayed cord clamping, formula feeding, kangaroo, mother care, novel corona virus, rooming-in, skin to skin care

\footnotetext{
*Address for correspondence: Sreenivas Karnati, MD, Department of Neonatology, M31, 9500 Euclid Ave, Cleveland, OH
} 


\section{Introduction}

Severe acute respiratory syndrome-coronavirus-2 (SARS-CoV-2), the virus responsible for the current novel coronavirus disease 2019 (COVID-19) pandemic, was first identified in Wuhan, Hubei, China in December 2019. In order to curb the pandemic, it is important to identify possible routes of transmission, including those between mother and infant. Pregnant women are a high-risk group, as such they are more likely to experience a more severe version of the disease if they become infected by SARS-CoV-2 [1]. They are also more likely to experience adverse pregnancy outcomes such as preterm birth, fetal growth restrictions, and neonatal death [2]. However, the risk of transmitting the virus from mother to infant through various delivery, feeding, and isolation methods is still unknown.

Past epidemics and pandemics have revealed variable rates and mechanisms of viral transmission from mother to infant. During both the 2003 SARS and Middle East respiratory syndrome (MERS) outbreaks in the 2010s, which were also caused by coronaviruses, researchers did not identify any cases of vertical transmission - babies born to SARS and MERS positive mothers were infection free [3]. Contrastingly, during the 2014 - 2015 Ebola epidemic, researchers found that vertical transmission from an acutely affected mother to her fetus is possible. The Ebola virus has also been detected in the breastmilk of mothers with the disease. As such, the CDC recommends against breastfeeding for women with confirmed Ebola infections and women who have recently recovered from the Ebola virus.

Current research on how SARS-CoV-2 is transmitted between mother-infant dyads is inconclusive. While it appears that in utero vertical transmission rates are low, a number of case reports and studies reveal that vertical transmission is possible. While transmission through breastmilk seems unlikely [4], studies have shown that the virus is sometimes detectable in breastmilk from infected mothers [5]. Further, it remains unclear whether the physiologic importance of skin-to-skin contact and delayed cord clamping for the infant and rooming in for the mother outweighs the risk of such practices for infants born to SARS-CoV-2 positive mothers.

Given the current evidence, it is difficult for studies to discern the safety of practices including breastfeeding. Therefore, the primary objective of this systematic literature review is to compare SARS$\mathrm{CoV}-2$ transmission rates between breast-fed infants and formula-fed infants. Our secondary objective is to determine the transmission rates of the disease in infants who roomed-in with their mothers, who have experienced delayed cord clamping, and who were given skin-to-skin care immediately after birth.

\section{Methods}

\subsection{Search strategy}

A search strategy was developed with a medical librarian to identify relevant literature. Electronic searches were performed in the Ovid MEDLINE, Ovid Embase, Cochrane Library, EBSCOhost CIN AHL Plus, Web of Science, and Scopus databases on November 19, 2020. The date range was limited from January 2019 to the date of the search with no limitation on language or publication type. Medical subject heading (MeSH) terms, other controlled vocabulary terms, and keywords related to Covid-19 infection, infant feeding techniques (e.g., breast/bottle feeding, weaning), and perinatal care for both mother and child were used in combination to retrieve relevant literature. The protocol for this review is registered in PROSPERO and the international prospective register of systematic reviews: CRD42020218722.

\subsection{Study selection}

We only included publications with full-texts available in English. We included studies which involved pregnant patients who tested positive for SARS-CoV2 by RT-PCR at the time of delivery with a viable baby or in the postpartum period. Neonates who had two SARS-CoV-2 tests, with the first being negative within 48 hours of birth, were included. Neonates more than one week old with a single positive RTPCR test were also included. All study designs except practice guidelines, literature reviews, and systematic reviews were included. We excluded non-human studies and studies that involved HIV positive pregnant patients. Any studies that did not report on primary outcomes of interest in perinatal practices (breast- or bottle-feeding) or secondary outcomes (kangaroo care or not, rooming-in or isolation, early or delayed cord clamping) were excluded.

Two independent reviewers (MC and SK) examined all titles and abstracts for relevance. Any disputes were resolved by vote of a third independent reviewer (CC). Three independent reviewers (MC, CC, and SK) then read and assessed all full-text articles for 
eligibility to be included in the qualitative review. Studies were grouped according to outcomes: (1) breast- vs. bottle-feeding, (2) kangaroo mother care vs. no skin-to-skin care, (3) rooming- in vs. isolation, and (4) early cord clamping (ECC) vs. delayed cord clamping (DCC).

\subsection{Data extraction}

Data were compiled into tables listing the first author, country, study design, number of included patients, and number in each intervention group.

In the studies deemed eligible for analysis, we included only patients for whom SARS-CoV-2 testing timeline and perinatal interventions were clearly reported. This means that the number of patients included in our study did not always equal the total number of patients included in a study. Within each outcome (breast/bottle-feeding, kangaroo care/not, rooming-in/isolation, and ECC/DCC), patients were grouped on the basis of SARS-CoV-2 positivity.

\subsection{Quality assessment}

The quality assessment was performed by 3 reviewers using the Newcastle-Ottawa Scale available at (http://www.ohri.ca/programs/clinical_epide miology/oxford.asp). Quality rating was determined based on (1) selectivity of study population, (2) comparability of groups, and (3) outcomes reported. Based on the conversions to AHRQ standards available at (https://www.ncbi.nlm.nih.gov/books/ NBK115843/bin/appe-fm3.pdf), studies were graded accordingly:

Good quality: 3 or 4 stars in selection domain and 1 or 2 stars in comparability domain and 2 or 3 stars in outcome/exposure domain

Fair quality: 2 stars in selection domain and 1 or 2 stars in comparability domain and 2 or 3 stars in outcome/exposure domain

Poor quality: 0 or 1 star in selection domain or 0 stars in comparability domain or 0 or 1 stars in outcome/exposure domain

\subsection{Statistical methods}

Given the nature of the studies included and their small sample sizes, a meaningful meta-analysis could not be done. However, we aggregated these data to perform a series of comparative analyses using Fisher's exact tests and evaluated the $p$-values to determine statistical significance of the findings. All analyses were done using SAS (version 9.4, The SAS Institute, Cary, NC) and a $p<0.05$ was considered statistically significant.

\section{Results}

\subsection{Study selection}

We identified 4893 records through database searches. After duplicates were removed, 788 studies remained for screening at the title and abstract level. Of these, 152 met our study's eligibility criteria and advanced to full-text review. 124 articles were then excluded for the following reasons: wrong study design $(n=65)$, inadequate SARS-CoV2 testing protocol $(n=26)$, wrong outcomes $(n=18)$, wrong language $(n=6)$, wrong patient population $(n=7)$, or wrong intervention $(n=2)$. The PRISMA diagram is presented in Fig. 1. The final qualitative and crude quantitative analysis included data from 28 studies.

\subsection{Quality assessment}

Information regarding quality assessment of studies included in this review are detailed in Table 1. Of 28 studies, 6 were rated as good quality, 1 as fair, and 21 as poor quality using the Newcastle-Ottawa Scale. The study designs included four prospective observational, one multicentric cohort, and three retrospective chart reviews, seven case series, and thirteen case reports. Despite some studies having a large patient population with a strong study design, inadequacy of follow-up testing for SARS-CoV-2 or poor documentation of perinatal practices resulted in low-grade scores for risk of bias assessment.

\subsection{Breast-feeding vs. bottle-feeding}

Breast- or bottle-feeding practices were clearly documented in 25 studies (Table 2). Any neonates who were fed from the mother's breast, including expressed maternal breast milk, were included in the breast-feeding group. Any neonates who received exclusively donor breast milk or formula were included in the bottle-feeding group. If intake was mixed, the neonate was included in the breastfeeding group. A total of 190 neonates were included in the breastfeeding category and 152 in the bottlefeeding category. 


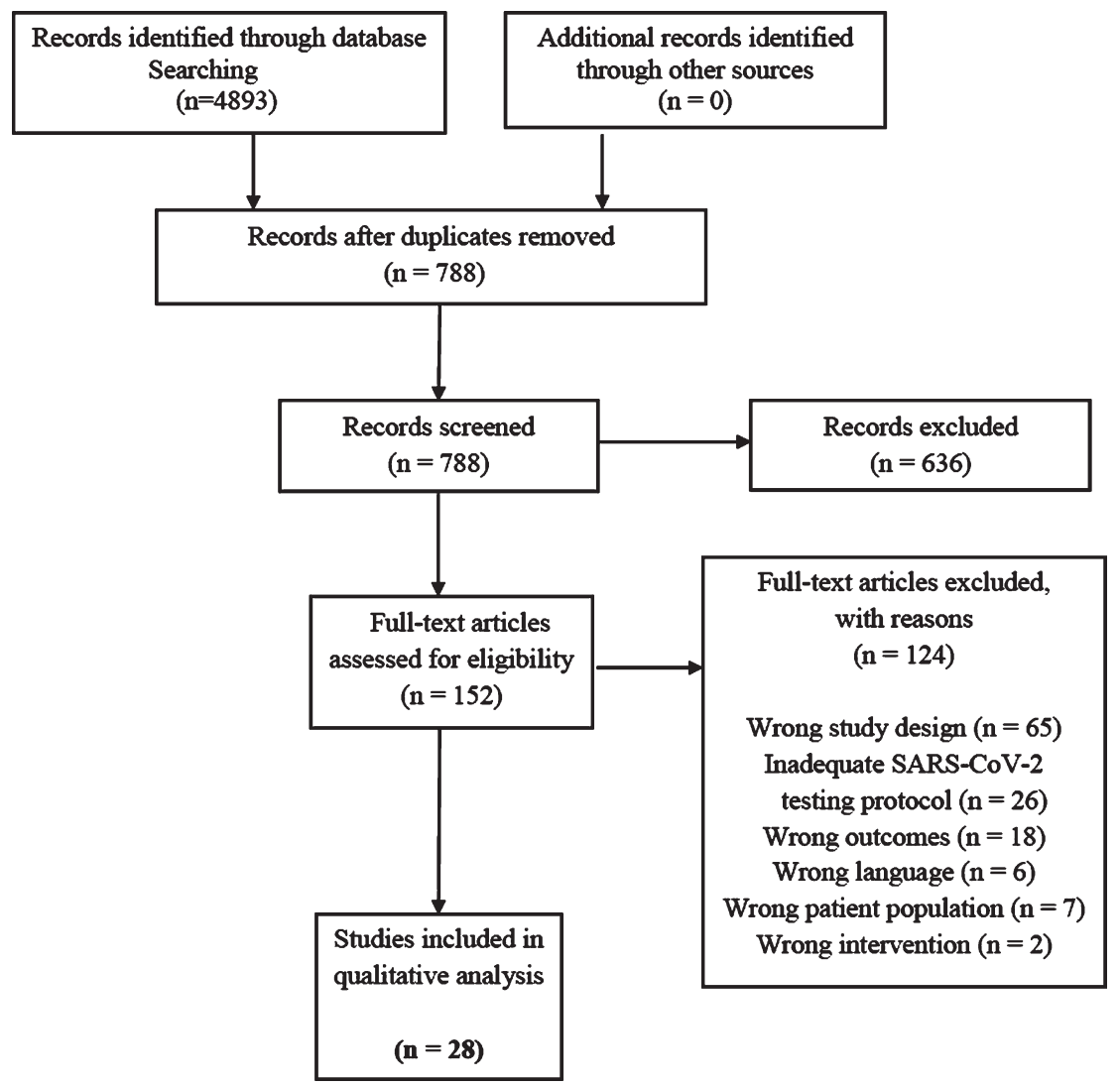

Fig. 1. PRISMA diagram showing number of studies identified, screened, assessed for eligibility, and included in the final analysis.

Table 1

Risk of bias assessment of included studies using Newcastle-Ottawa Scale

\begin{tabular}{lll}
\hline Quality & Type of study & First author and country \\
\hline Good (6) & Case series & Romagano- USA [16] \\
& Prospective observational & Salvatore-USA [6], Bertino- Italy [10], \\
& Popofsky-USA [12] \\
& Retrospective chart review & Griffin-USA [19] \\
Multicentric cohort study & Oncel-Turkey [7] \\
Fair $(\mathrm{N}=1)$ & Case series & Biasucci -Italy [8] \\
Poor $(\mathrm{N}=21)$ & Case reports & Sinelli-Italy [15], Mochizuki-Japan [17], \\
& & Alzhagal-Jordan [20], Bastugi-Turkey [21], \\
& & Olcese-Peru [22], Ronnje- Sweden [23], \\
& Dong-China [29], De Socio-Italy [30], \\
& Lang-China [31], Perrone-Italy [32], Lugli-Italy \\
& [33], Makwe-Nigeria [34], Sagheb-Iran [35] \\
& Case series & Costa- Italy [9], Anand- India [14], Pissarra-Portugal \\
& [18], Zheng-China [24], Salvatori- Italy [27] \\
& Prospective observational & Mejia-Jimenez - Spain [25] \\
Retrospective chart review & Kalamdani-India [11], Zhang-China [13] \\
\hline
\end{tabular}

Many studies reported hospital protocols that allowed for breastfeeding between mother-infant dyads where the mother was COVID-19 positive, but required hand hygiene and mask wearing during feeding [6-9]. Despite these precautions, the total number of neonates who tested positive in the breastfeeding group was 22 of 190 (11.5\%). This was significantly higher than the positivity rate in the bottle-fed group, which was $2.63 \%$ or 4 of 152 infants $(p=0.0006)$.

From Bertino et al., a prospective observational study, we excluded 3 patients who tested positive for SARS-CoV-2 within 48 hours of birth and 
Table 2

Breast vs. bottle-fed infants

\begin{tabular}{lccccc}
\hline Type of study & $N$ & BF+ & BF- & Bottle+ & Bottle- \\
\hline Case reports [15, 17, 20-23, 30, 32-35] & 11 & 2 & 3 & 1 & 5 \\
Case series [8, 9, 14, 16, 18, 24, 27] & 36 & 6 & 13 & 0 & 17 \\
Prospective Observational [6, 10, 12] & 168 & 1 & 103 & 0 & 64 \\
Retrospective chart review [11, 13] & 13 & 13 & & & 62 \\
Multi center cohort [7] & 114 & & 49 & 3 & $\mathbf{1 4 8}$ \\
Total & $\mathbf{3 4 2}$ & $\mathbf{2 2}$ & $\mathbf{1 6 8}$ & $\mathbf{4}$ & $\mathbf{1 4 8}$ \\
\hline
\end{tabular}

$\mathrm{BF}=$ breast-fed infants. Bottle $=$ bottle-fed infants. $+=$ positive for SARS-CoV-2 by RT-PCR.$-=$ negative for SARS CoV-2 by RT-PCR. $N=$ number of infants.

subsequently tested negative at all other time points for suspicion of vertical transmission [10].

Kalamdani et al., a large retrospective chart review of 185 exclusively breastfed neonates, did not have the resources to test all infants twice for SARS-CoV2 [11]. We included only patients who had repeat testing. Accordingly, from Salvatore's observational cohort study we could only include 74 of 82 patients for whom follow-up testing was clearly completed [6].

For Oncel et al., a multicentric cohort study, SARSCoV-2 testing was unknown for 5 infants [7]. We thus excluded an equal number of patients from each feeding group. One patient in the bottle-feeding group had an inconclusive test result and was also excluded. Similarly, Popofsky et al. did not clearly document feeding practices for one patient; we therefore excluded one neonate from both groups [12].

Sensitivity analysis, after excluding studies with small sample size $(<5)$, showed SARS-CoV-2 positivity rates of $8.43 \%(15 / 178)$ for the breast fed group and $2.13 \%$ (3/141) for the infants fed by other modes $(p=0.0153)$.

Majority of the infants included were either term or late preterm at birth. Almost all of the infants who were tested positive for SARS-CoV-2 were asymptomatic. Two neonates developed low grade fevers but without respiratory symptoms [11, 13]. Single preterm infant who was born at 32 weeks had respiratory symptoms, which were presumed to be related to prematurity rather than SARS-CoV-2 infection [14].

\subsection{Kangaroo care vs. separation}

Only 12 studies clearly documented kangaroo care following delivery. Key phrases which indicated kangaroo care occurred were "immediate skin-to-skin contact" and "immediate bonding" $[8,15]$.

A number of studies documented immediate separation of the baby from mother upon delivery. Key phrases used to determine inclusion in the no kangaroo care group included "strict isolation measures," "separated immediately after delivery," and documentation of the first time mother held the baby [16-18]. We did not assume that infants cared for in the NICU were in the no kangaroo care group. Only 4 of 8 patients from Romagano et al.'s study were included, as 1 patient was undelivered, 1 patient did not complete follow-up testing, and 2 patients were not tested within 48 hours of birth [16].

Of the 16 neonates included in the kangaroo care group, 4 tested positive for SARS-CoV-2 (25\% positivity rate). In total, 2 of 22 patients in the no kangaroo care group tested positive for SARS-CoV-2 (9.09\%). There was no significant difference in positivity rate between the two groups $(p=0.2170)$.

All the infants who were tested positive for SARS$\mathrm{CoV}-2$ remained asymptomatic.

\subsection{Rooming-in vs. isolation}

A total of 23 studies (Table 3) included information about rooming-in practices during hospital stay. Phrases which indicated rooming-in were "no isolation," and "roomed-in" [8, 10-12]. We also included in the roomed-in category any patients who were discharged home with mom and later readmitted for COVID evaluation [11].

Key words which indicated isolation protocols included "kept in a separate room," "immediate separation," "isolation and separation," and "followed in isolation rooms" [7, 19-21].

Oncel et al. documented 11 cases in which infants were kept at a distance of 2 meters from the mother; due to ambiguity we decided to exclude these patients [7]. Another 6 were cared for "by family members in a separate room," but we could not determine if this implied no visitation by the mother and also excluded them. An additional 1 patient was deemed a false positive and 5 were untested; 102 of 125 from Oncel et al. were thus included [7]. In Griffin's retrospective review, only 13 of 78 cases had clear testing 
Table 3

Rooming in vs. isolation

\begin{tabular}{|c|c|c|c|c|c|}
\hline Type of study & $N$ & RI+ & RI- & Iso+ & Iso- \\
\hline Case reports $[15,17,20-23,29-31,33-35]$ & 12 & 1 & 1 & 2 & 8 \\
\hline Case series $[8,18,24,27]$ & 28 & 4 & 11 & & 13 \\
\hline Prospective Observational $[6,10,12]$ & 235 & 2 & 71 & & 162 \\
\hline Retrospective chart review $[11,13,19]$ & 26 & 13 & & & 13 \\
\hline Multi center cohort [7] & 102 & & & 3 & 99 \\
\hline Total & 403 & 20 & 83 & 5 & 295 \\
\hline
\end{tabular}

$\mathrm{RI}=$ roomed-in infants. Iso = isolated infants. $+=$ positive for SARS-CoV-2 by RT-PCR. $-=$ negative for SARSCoV-2 by RT-PCR. $N=$ number of infants.

documentation for both mother and baby; all were initially isolated upon delivery [19].

The rooming-in group totaled 103 neonates, 20 of whom tested positive for SARS-CoV-2 (19.4\%). The isolation group consisted of 300 patients, 5 of whom tested positive for the virus $(1.67 \%)$. There was a significant difference in positivity rate between the two groups $(p<0.0001)$.

On further sensitivity analysis, after excluding case reports and case series with $<5$ cases, SARS-CoV2 positivity rate in rooming-in group was $15.45 \%$ $(15 / 97)$, while in isolation group it was $1.04 \%(3 / 289)$ $(p<0.0001)$.

None of the infants tested positive for SARS-CoV2 exhibited any symptoms.

\subsection{Delayed vs. early cord clamping}

Five studies clearly documented early or delayed cord clamping practices (ECC and DCC). Four were case reports or case series [15, 22-24]. MejiaJimenez et al. was the only study to describe a large number of cases [25].

For Delayed vs Early Cord Clamping, SARSCoV-2 positivity rate in DCC group was $3.62 \%$ $(5 / 138)$, while that in ECC group was $0.90 \%(2 / 222)$ $(p=0.1116)$.

Similar to results of previous practices, infants were asymptomatic despite being tested positive for the virus.

\section{Discussion}

Our final inclusion criteria required a negative SARS-CoV-2 test within 48 hours of birth in order for there to be some degree of certainty that the infant did not contract the virus from vertical transmission. Umbilical cord clamping practice was an exception to this as the positivity rates for the virus may reflect vertical transmission. For this, we included the infants who were tested within 48 hours of birth. In addition, for neonates still within their postpartum hospitalization period, a second SARS-CoV-2 test was required to demonstrate persistent negative status or newly positive status as a result of horizontal transmission. Many (26 of 152) studies whose full-texts were reviewed did not meet these criteria for any of their patients, and a number of studies which were included in final analysis only met testing requirements for a portion of their sample. Due to this issue, the total number of patients we were able to include in our quantitative analysis was limited.

We propose that an ideal testing timeline for neonates born to SARS-CoV-2 positive mothers include at least two tests: one before 48 hours of life and a second at least two days later, within the first 14 days of life. It is important to continue testing protocols even if a neonate initially tests negative at birth. Kalamdani et al. were only able to re-test those infants who initially tested positive or developed symptoms [11]. Thus any asymptomatic neonates of breastfeeding mothers who experienced horizontal viral transmission went undetected. Since the long-term effects of SARS-CoV-2 remain unknown, early diagnosis via consistent testing is critical to ensure that a neonate's history of prior illness is appropriately documented in their medical chart in case of future complications.

Despite the heterogeneity of testing protocols and few studies meeting our inclusion criteria, the comparison between breast and bottle-feeding infants showed a significant difference in the positivity rate of the two groups: $11.5 \%$ to $2.63 \%$. This may insinuate that bottle-feeding is safer than breast-feeding in mother-infant dyads where the mother is known to be SARS-CoV-2 positive. However, of the 13 studies that tested breastmilk for SARS-CoV-2 RNA, 9 of them did not detect viral particles in any samples [7, $9,10,26,27]$. Bertino et al. tested 14 milk samples for SARS-CoV-2 RNA, and only one returned positive - that neonate later tested positive for the virus, 
but had been put to skin and roomed-in without any precautions [10]. Salvatori et al. tested 2 milk samples, both negative, but the neonates tested positive [27]. This suggests that the increased transmission rate by SARS-CoV-2 positive breast-feeding mothers may not be due to viral load in the milk, but instead caused by other means such as respiratory droplet transmission due to poor hand hygiene or poor mask adherence during feeds.

If proximity to SARS-CoV-2 positive mothers increases the rate of horizontal viral transmission, we would also expect there to be an increased in positivity rate among dyads participating in kangaroo care compared to those infants who were not allowed postpartum skin-to-skin contact with mothers. Though our analysis did find a greater number of positive cases among kangaroo care-treated neonates (4/16), this was not a statistically significant difference compared to those who did not receive skin-to skin care $(2 / 22)$. The small sample size of each group limited the power of this analysis. Further studies in environments enforcing rigorous hygiene and masking protocols among kangaroo mothers should be completed to better elucidate the relative risk of horizontal transmission with skin-to-skin contact. Kangaroo care is considered standard practice and essential to maternal-infant bonding; our study cannot conclude that there is an increased risk of SARS-CoV-2 transmission with this practice [28].

The results of our analysis of rooming-in and isolated infants suggest that prolonged proximity to a SARS-CoV-2 positive mother increases the likelihood of horizontal transmission. Significantly more (19.4\%) number of roomed-in neonates contracted the virus, while only $1.67 \%$ of infants who underwent isolation tested positive $(p<0.0001)$. This may be due to prolonged exposure to respiratory droplets and aerosolized virions as a result of inconsistent masking protocols or inadequate air circulation systems. Maternity wards are generally not negative-pressure environments or designed for frequent air changes, which are valuable engineering controls that help contain the spread of SARS-CoV-2 in the presence of an actively infectious individual. Though maternalinfant bonding is a crucial aspect of perinatal care, the results of this analysis caution against prolonged proximity between an infectious mother and her infant.

Our analysis on early and delayed cord clamping practices was similarly limited by a lack of robust studies which included proper testing timeline and documentation of cord clamping for all patients. The overall analysis shows no statistical difference in positivity rate of the two groups $(3.6 \%$ DCC and $0.9 \%$ ECC). The beneficial health effects of delayed cord clamping for improving neonatal outcomes are widely recognized. This analysis supports the hypothesis that delayed cord clamping does not present any added risk of SARS-CoV-2 transmission from a positive mother to infant, though more robust studies with representative samples should be conducted.

The heterogeneity of study design and the inconsistency of SARS-CoV-2 testing protocols across studies additionally presented a challenge for analysis. Overall, the vast majority of studies included in this review were case reports or case studies (23/28). This was particularly notable in the cohort included in the DCC vs. ECC comparison, wherein $80 \%$ of the studies contained 4 or fewer patients. The small sample sizes and poor representativeness of their samples made these weak studies for analysis. This was not a meta-analysis and the quantitative results should be treated with scrutiny.

Despite some of the results from this review (breast feeding and rooming-in) point to an increased positivity rate, it did not translate in to an increased number of symptomatic infants. As most of these infants remained asymptomatic, the benefits of breast milk and bonding with the mothers should take precedence over formula feeding and separation wherever appropriate. These findings reiterate the need for standardized guidelines and protocols. At the same time, common practices to prevent spread of SARS-COV-2 like social distancing, use of face masks and thorough hand washing should be reinforced.

\section{Conclusions}

The literature around SARS-CoV-2 horizontal transmission and perinatal practices remains sparse and riddled with protocol inconsistencies. From the available evidence, there appears to be an increased risk of transmission of SARS-CoV-2 to neonates from breast feeding and rooming -in practices. Infants however remained asymptomatic. No increased viral transmission was observed in infants who received delayed cord clamping or early skin to skin care. More studies with pre-established testing protocols and clear documentation of perinatal practices should be conducted to better elucidate the risk of horizontal transmission to infants among mothers who are SARS-CoV-2 positive. 


\section{Conflict of interest}

The authors declare that they have no commercial or financial relationships that could be construed as a potential conflict of interest.

\section{References}

[1] https://www.cdc.gov/coronavirus/2019-ncov/need-extraprecautions/people-with-medical-conditions.html.

[2] Lam CM, Wong SF, Leung TN, Chow KM,Yu WC, Wong TY, Lai ST. A case-controlled study comparing clinical course and outcomes of pregnant and non-pregnant women with severe acute respiratory syndrome. BJOG. 2004;111:771-4.

[3] Schwartz DA, Graham AL. Potential maternal and infant outcomes from coronavirus 2019-NCOV (SARS-CoV2) infecting pregnant women: Lessons from SARS, MERS, and other human coronavirus infections. Viruses. 2020;12(2):1-16.

[4] Chen H, Guo J, Wang C, Luo F, Yu X, Zhang W, et al. Clinical characteristics and intrauterine vertical transmission potential of COVID-19 infection in nine pregnant women: a retrospective review of medical records. Lancet. 2020;395:809-15

[5] Centeno-Tablante E, Medina-Rivera M, Finkelstein JL, Rayco-Solon P, Garcia-Casal MN, Rogers L, et al. Transmission of SARS-CoV-2 through breast milk and breastfeeding: A living systematic review. Ann N Y Acad Sci. 2021;1484(1):32-54.

[6] Salvatore CM, Han JY, Acker KP,Tiwari P, Jin J, Brandler $\mathrm{M}$, et al. Neonatal management and outcomes during the COVID-19 pandemic: An observation cohort study. Lancet Child Adolesc Heal. 2020;4:721-7.

[7] Oncel MY, Akın IM, Kanburoglu MK, Tayman C, Coskun $\mathrm{S}$, Narter F, et al. A multicenter study on epidemiological and clinical characteristics of 125 newborns born to women infected with COVID-19 by Turkish Neonatal Society. Eur J Pediatr. 2021;180(3):733-42.

[8] Biasucci G, Cannalire G, Raymond A, Capra ME, Benenati B, Vadacca G, et al. Safe perinatal management of neonates born to sars-cov- 2 positive mothers at the epicenter of the italian epidemic. Front Pediatr. 2020;8:1-6.

[9] Costa S, Posteraro B, Marchetti S, Tamburrini E, Carducci B, Lanzone A, et al. Excretion of SARS-CoV-2 in human breast milk. Clin Microbiol Infect. 2020;26:1430-2.

[10] Bertino E, Moro GE, De Renzi G, Viberti G, Cavallo $\mathrm{R}$, Coscia A, et al. Detection of SARS-CoV-2 in Milk From COVID-19 Positive Mothers and Follow-Up of Their Infants. Front Pediatr. 2020;8:1-6.

[11] Kalamdani P, Kalathingal T, Manerkar S, Mondkar J. Clinical profile of sars-cov-2 infected neonates from a tertiary government hospital in Mumbai, India. Indian Pediatr. 2020;57(12):1143-6.

[12] Popofsky S, Noor A, Leavens-Maurer J, QuintosAlagheband ML, Mock A, Vinci A, et al. Impact of maternal severe acute respiratory syndrome coronavirus 2 detection on breastfeeding due to infant separation at birth. J Pediatr. 2020;226:64-70.

[13] Zhang ZJ, Yu XJ, Fu T, Liu Y, Jiang Y, Yang BX BY. Novel coronavirus infection in newborn babies aged $<28$ days in China. Eur Respir J. 2020;55(6):2000697.
[14] Anand P, Yadav A, Debata P, Bachani S, Gupta N, Gera R. Clinical profile, viral load, management and outcome of neonates born to COVID 19 positive mothers: A tertiary care centre experience from India. Eur J Pediatr. 2021;180(2):547-59.

[15] Sinelli M, Paterlini G, Citterio M, Di Marco A, Fedeli T, Ventura ML. Early neonatal SARS-CoV-2 infection manifesting with hypoxemia requiring respiratory support. Pediatrics. 2020;146(1).

[16] Romagano MP, Guerrero K, Spillane N et al. Perinatal outcomes in critically ill pregnant women with coronavirus disease 2019. Am J Obs Gynecol MFM. 2020;2(3):100151.

[17] Mochizuki J, Nakamura M, Iwahata S, Nishijima J, Ito T, Wada T, et al. First report in Japan of a delivery of a woman with the 2019 novel coronavirus disease. J Obstet Gynaecol Res. 2021;47(1):407-10.

[18] Pissarra S, Rosário M, Moucho M, Soares H. Perinatal management of SARS-CoV-2 infection in a level III University Hospital. J Matern Neonatal Med. 2020;0(0):1-4.

[19] Griffin I, Benarba F, Peters C, Oyelese Y, Murphy T, Contreras D, et al. The impact of COVID-19 infection on labor and delivery, newborn nursery, and neonatal intensive care unit: Prospective observational data from a single hospital system. Am J Perinatol. 2020;37(1):1022-30.

[20] AlZaghal LA, AlZaghal N, Alomari SO, Obeidat N, Obeidat B, Hayajneh WA. Multidisciplinary team management and cesarean delivery for a Jordanian woman infected with SARS-COV-2: A case report. Case Reports Women's Heal. 2020;27:e00212.

[21] Bastug A, Hanifehnezhad A, Tayman C, Ozkul A, Ozbay O, Kazancioglu S, et al. Virolactia in an asymptomatic mother with COVID-19. Breastfeed Med. 2020;15(8):488-91.

[22] Campodónico Olcese L, Paredes Salas JR, Campodónico Olcese D, Chang Vargas C, Acuña Barrueto L, Marchena Arias J. Management of eutocic delivery in a patient with COVID-19 in Lima, Peru. Rev Peru Ginecol y Obstet. 2020;66(2):101-4.

[23] Ronnje L, Länsberg J-K, Vikhareva O, Hansson S, Herbst A, Zaigham M. Complicated COVID-19 in pregnancy: A case report with severe liver and coagulation dysfunction promptly improved by delivery. BMC Pregnancy Childbirth. 2020;8:1-8.

[24] Zheng T, Guo J, He W, Wang H, Yu H, Ye H. Coronavirus disease 2019 (COVID-19) in pregnancy: 2 case reports on maternal and neonatal outcomes in Yichang city, Hubei Province, China. Medicine (Baltimore). 2020; 99(29):e21334.

[25] Mejía Jiménez I, Salvador López R, García Rosas E, Rodriguez de la Torre I, Montes García J, Cruz Conty $\mathrm{M}$, et al. Umbilical cord clamping and skin-to-skin contact in deliveries from women positive for SARS-CoV-2: a prospective observational study. BJOG An Int J Obstet Gynaecol. 2020;2019:1-8.

[26] Dong Y, Chi X, Hai H, Sun L, Zhang M, Xie WF, et al. Antibodies in the breast milk of a maternal woman with COVID-19. Emerg Microbes Infect. 2020;9(1):1467-9.

[27] Salvatori G, De Rose DU, Concato C, Alario D, Olivini N, Dotta A, et al. Managing COVID-19-positive maternalinfant dyads: An Italian experience. Breastfeed Med. 2020;15(5):347-8.

[28] Browne J. Early relationship environments: Physiology of skin-to-skin contact for parents and their preterm infants. Clin Perinatol. 2004;31(2):287-98.

[29] Dong L, Tian J, He S, Zhu C, Wang J, Liu C, Yang JM. Possible vertical transmission of SARS-CoV-2 from an infected 
mother to her newborn. JAMA [Internet]. 2020;323: 1846-8.

[30] De Socio GV, Malincarne L, Arena S, Troiani S, Benedetti S, Camilloni B, et al. Delivery in asymptomatic Italian woman with SARS-CoV-2 infection. Mediterr J Hematol Infect Dis. 2020;12(1):2-4.

[31] Lang G jing, Zhao H. Can SARS-CoV-2-infected women breastfeed after viral clearance? J Zhejiang Univ Sci B. 2020;21(5):405-7.

[32] Perrone S, Giordano M, Meoli A, Deolmi M, Marinelli F, Messina $\mathrm{G}$, et al. Lack of viral transmission to preterm newborn from a COVID-19 positive breastfeeding mother at 11 days postpartum. J Med Virol. 2020;92(11):2346-7.
[33] Lugli L, Bedetti L, Lucaccioni L, Gennari W, Leone C, Ancora G. An uninfected preterm newborn inadvertently fed SARS-CoV-2 - positive breast milk. 2021;147(4):2-6.

[34] Makwe CC, Okunade KS, Rotimi MK, Ekor OS, Oyeleke OG, Bello QO, et al. Caesarean delivery of first prediagnosed COVID-19 pregnancy in Nigeria. Pan Afr Med J. 2020;36:100.

[35] Sagheb S, Lamsehchi A, Jafary M, Atef-Yekta R, Sadeghi K. Two seriously ill neonates born to mothers with COVID-19 pneumonia- a case report. Ital J Pediatr. 2020;46(1):1-6. 